\title{
REMLABNET - User Experience and Mixed Reality Continuum
}

\author{
https://doi.org/10.3991/ijoe.v14i02.7651 \\ Tomas Komenda $\left({ }^{\varpi}\right)$, Franz Schauer \\ Tomas Bata University in Zlin, Zlin, Czech Republic \\ komendatomas@seznam.cz
}

\begin{abstract}
Our recent research in remote laboratory management systems (REMLABNET-www.remlabnet.eu) deals with questions such as how to make the user experience stronger and how to help users understand complex phenomena behind remote experiments and the laws of physics governing the experiment. At our current stage of technological development, we have both sufficiently powerful hardware and software to create an impressive virtual user interface which could be a help to this mission. An extended mixed reality taxonomy for remote physical experiments was proposed to identify goals of the future REMLABNET research and development. The first part of this paper describes classes of taxonomy and reasons why they were set up in this way. The second part mentions the chosen method of our research and our current progress.
\end{abstract}

Keywords-remote physical experiment, mixed reality, virtual reality, user experience

\section{Introduction}

REMLABNET (version 2017) is a Remote Laboratory Management System (RLMS) which allows combining the research world of physics with modern elements of information technologies. The system provides remote control of both real experiments and simulation of experiments via a web interface and offers possibilities to process, observe, record and study results [1], [2]. These remote experiments include a server with a set of physical objects of study (rigs). Distance education offers a possibility to mix real objects with simulated ones and to create hybrid rigs (or to replace the whole process with simulation)[3]. The disadvantage is the absence of users in the laboratory. It is necessary to broadcast current events from the laboratory to users. Observation of both the rig and the measuring process is an essential part that in many cases leads to a real understanding of the task. The quality of such interaction has a major impact on the user's learning curve and their satisfaction with RMLSs. REMLABNET now provides a web interface with control elements and 2-D video streaming. We decided to extend REMLABNET and start a research project towards user interface and user experience. Rapid development and technological progress in virtual reality (VR) devices (e.g. smart glasses and VR helmets) bring new 
possibilities that RLMSs can offer to their users. VR has been linked to remote experiments for three decades [4]. One of the primary goals of virtual reality is to provide immersive environments that transfer participants from real life to a virtual one. This idea has the potential to improve remote physical experiments by providing a rich sense of interactive control and immersion in the environment. VR became a mature discipline combining computer graphics, computer vision, object recognition, simulation, artificial intelligence, and data mining. It also contains a lot of subcategories, e.g. augmented reality (AR), augmented virtuality (AV), mediated reality (MR), simulated reality (SR), and others. But to date, VR and RLMSs have not been well integrated because of a lot of technical and logical limitations [5]. Due to this complexity, it is an imperative to evaluate the risks and benefits of such a development. Our first steps were to identify REMLABNET in a mixed reality continuum and classify possible methods of research and subsequent development. For the purposes of REMLABNET and common development of remote physical experiments, we extend standard mixed reality taxonomy [8]. The first part of this paper describes classes of taxonomy and reasons why they were set up in this way. The second part mentions the chosen way of development and our current progress.

\section{$2 \quad$ Mixed reality taxonomy}

The taxonomy counts in important aspects and trends of modern RLMSs and remote-driven physical experiments, which REMLABNET is [2],[6]. On the other hand, we do not aim to cover the needs of more complex and extensive remote laboratory systems that combine multiple disciplinary approaches [7]. We proposed four classes based on user's devices (Table 1 and Figure 1). Current technical maturity, accessibility, price, and backward compatibility with current systems were taken into account.

These classes do not cover all devices and their combinations (e.g. smartphones, tablets, smart lenses, holograms, and various types of VR helmets). It is obvious that a monitor can be replaced with a tablet and smart glasses with a smartphone. For REMLABNET purposes, we chose EPSON Moverio BT- $300{ }^{1}$ (smart glasses) as a representative of Class 2. As a representative of Class 3, we decided to use a combination of Samsung Galaxy S7 and Samsung Galaxy Gear ${ }^{2}$ together with $180^{\circ}$ wideangle 3-D LucidCam ${ }^{3}$.

\footnotetext{
${ }^{1}$ EPSON Moverio BT-300 Smart Glasses (AR/Developer Edition). Si-OLED display (0.43" wide panel, $1280 \times 720 \times$ RGB, virtual screen size $\left.80^{\prime \prime}\right)$, a high-resolution camera (5 million pixels), Intel Atom chipset, Android 5.1, price about $\$ 800$ (April 2017). https://tech.moverio.epson.com/en/bt-300/ ${ }^{2}$ Samsung Galaxy Gear VR and Samsung Galaxy S7 Edge. 2560 x 1440 pixel Super AMOLED, $96^{\circ}$ field of view, $60 \mathrm{~Hz}$, price about $\$ 1000(\$ 900+\$ 100$, April 2017).

http://www.samsung.com/uk/smartphones/galaxy-s7/overview/, http://www.samsung.com/global/galaxy/gear-vr/

${ }^{3}$ LucidCam 3-D VR camera (prototype). 4K-30fps/eye videos, wifi supported for the live stream, the range of view $180^{\circ} \times 180^{\circ}$, price about $\$ 500$ (April 2017). https://lucidcam.com
} 
Table 1. Proposed Classes

\begin{tabular}{|c|l|l|}
\hline Class & \multicolumn{1}{|c|}{ Device } & \multicolumn{1}{c|}{ Short Description } \\
\hline 0. & $\begin{array}{l}\text { A laboratory experiment or local computer-driven experiment. There is no } \\
\text { remote access here. The user has to be present in the laboratory. This class is } \\
\text { defined by the generally mixed reality taxonomy and is not a subject of this } \\
\text { paper. }\end{array}$ \\
\hline 1. & Monitor, display & $\begin{array}{l}\text { A laboratory experiment is remotely controlled. Events and results are ob- } \\
\text { served on a standard monitor or display (our current stage in REMLABNET). }\end{array}$ \\
\hline 2. & $\begin{array}{l}\text { augmented reality } \\
\text { glasses (transparent } \\
\text { and lightweight VR } \\
\text { headsets) }\end{array}$ & $\begin{array}{l}\text { This class is a superset of Class 1. Smart glasses allow seeing data displayed } \\
\text { on a standard monitor through the glasses. Glasses bring an AR extension that } \\
\text { in the laboratory, and other users (e.g. 3-D mirroring of the rig, video chat with } \\
\text { other users, collaborative learning, sharing of the results). This class guaran- } \\
\text { tees a backward compatibility with Class 1 (it is possible to take the glasses off } \\
\text { and use RLMS like Class 1). }\end{array}$ \\
\hline 3. & $\begin{array}{l}\text { Virtual reality } \\
\text { headset (helmet) }\end{array}$ & $\begin{array}{l}\text { This class is a superset of Class 2; however, this class is not backward compat- } \\
\text { ible with Class 1 or Class 2. There is no possibility of seeing the monitor } \\
\text { through the headset. It is possible to take the headset off and use the monitor, } \\
\text { but not both at the same time. A wide-angle stereoscopic camera can stream } \\
\text { real events from the laboratory. This streamed scene can be extended by a } \\
\text { hybrid or fully virtual object. In extreme cases, the whole scene can be entirely } \\
\text { virtual. }\end{array}$ \\
\hline
\end{tabular}

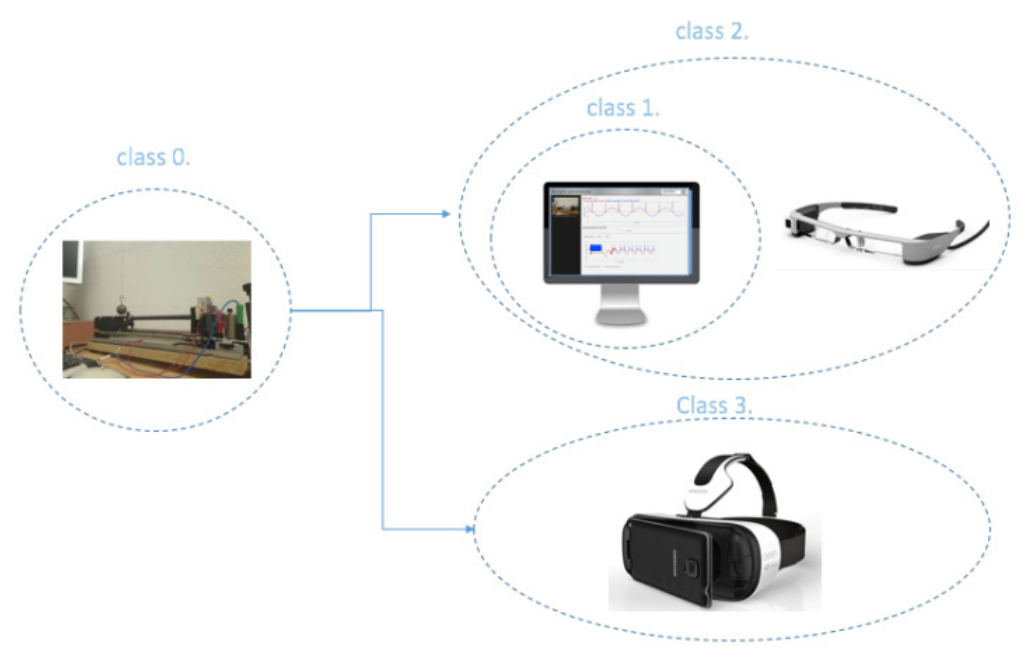

Fig. 1. Proposed class diagram
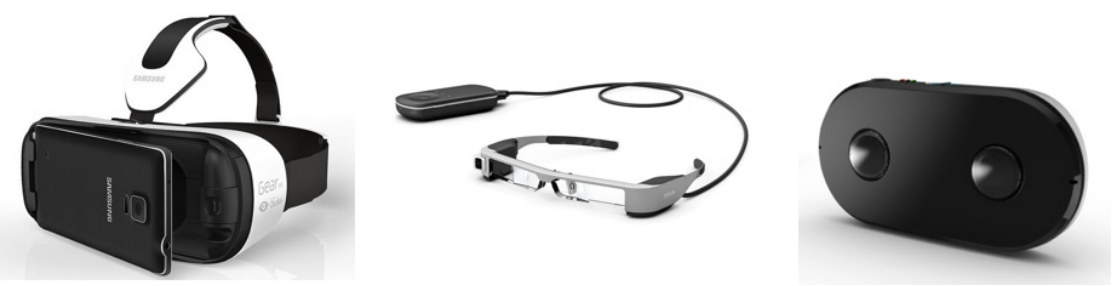

Fig. 2. Samsung Galaxy Gear (left), EPSON Moverio BT-300 (middle) and LuciCam (right) 
To improve user's experience, it is important to identify the level of utilization of the system into relevant classes. For these objectives to be achieved, we can use $\mathrm{S}$. Mann's mixed reality continuum [8]. Figure 3 (on the left) shows a two-dimensional continuum whose horizontal axis describes the degree of virtuality and vertical axis completes the degree of mediality. However, the user experience classification related to remote physical experiments requires more. RLMSs users remotely control and observe some events processed in the laboratory. Let's assume that a user is carrying out measurement of a simple pendulum. The rig is physically present in the laboratory. A simple pendulum is moving. The results are taken via sensors. The user can watch a video stream of events. In this case, the situation is categorized as real reality (RR). If the video stream is complemented with the information about results (charts, numbers, tables), the RR is mediated. However, it is possible not to stream video and show virtual models of the rig (2-D or 3-D) instead, and the events in the laboratory can be demonstrated on these models (there can be many reasons for this attitude, e.g. high network latency, fault of video devices, etc.). In this case, the user observes an entirely virtual model which mirrors real events of the rig. The classification is mediated virtual reality. It does not matter whether the user uses a common monitor or display. The recent trend in remote physical experiments is to combine or completely replace physical rigs (or their parts) with simulation [9]. This innovation is based on the idea of Simulation-based Learning (SBL). Due to this, mixed continuum has been extended into a three-dimensional space (Figure 3 - right). This space is not binary. Instead, it is more of a continuum, with many variations that are possible between the two extremes. For the sake of simplicity and clarity, however, the space is divided into discrete quadrants. Any quadrant can be perceived as a discrete point (it is possible to reach this stage-for purposes of future REMLABNET development we chose this approach) or as a continuum with a potentially more detailed classification.
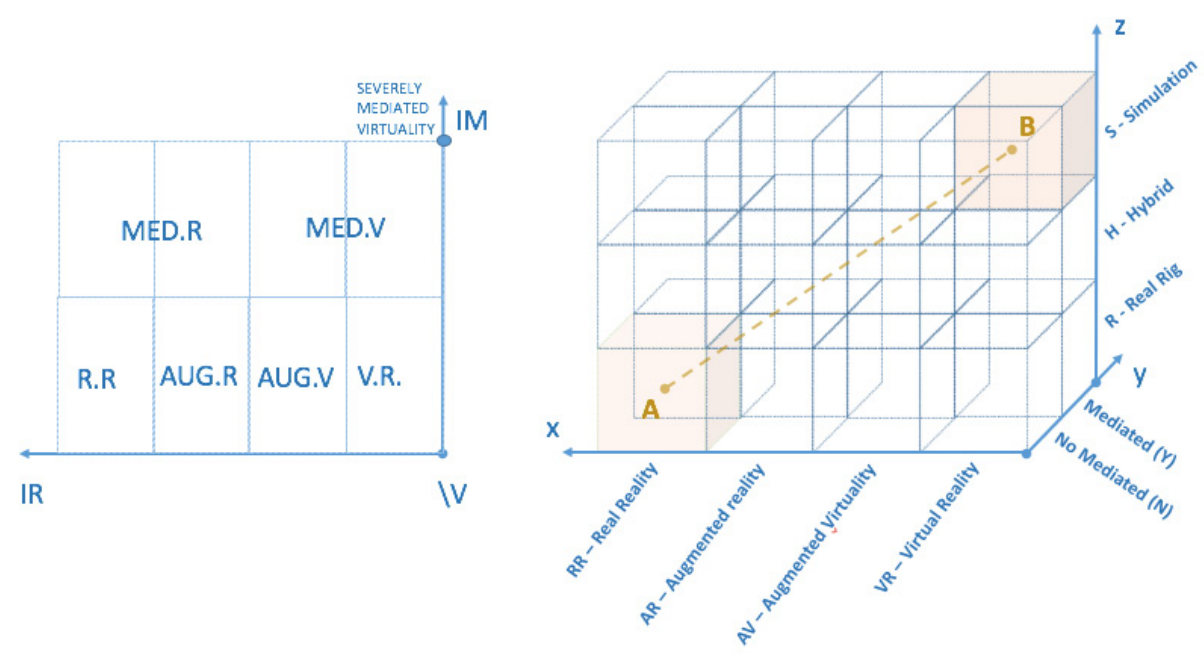

Fig. 3. Mixed reality continuum (left) and its extended version for remote physical experiments (right) 
We proposed a classification based on the class number and the relevant quadrant (CLASS.X.Y.Z). We can see two extremes in this taxonomy. 0.RR.N.R (Class 0 and a quadrant marked as $A$ in Error! Reference source not found..) would be a local experiment (observed by user's own eyes) running at the real rig. 1.RR.N.R (Class 1) would be a remote experiment observed via 2-D video stream (watched on a common monitor or display). On the other hand, 3.VR.Y.S (Class 3 and a quadrant marked as $B$ in Error! Reference source not found..) would be a completely simulated experiment (observed via a VR headset consisting only from virtual elements), which is so realistic that users are not able to decide whether they are carrying out the measurement via simulation or they are observing a mirroring of the real rig. In the most extreme point of this stage, this remote experiment would be very close to the idea of simulated reality [10].

\section{REMLABNET and the extended taxonomy}

For a long time, REMLABNET was classified as 1.RR.Y.R. It was possible to control and observe only real rigs via a web interface (HTML, JavaScript) [1]. However, students sometimes have problems understanding the complex phenomena behind remote experiments and the laws of physics governing these experiments. To provide insight into the physics of a certain phenomenon, a mixed multiparameter simulation, working with analytical formulations of the respective law, can be of great help. For this purpose, we designed a new module and implemented it into the remote experiment software, enabling simulations embedding into remote experiments outputs [1].

According to the proposed taxonomy, the embedded simulation moved REMLABNET into 1.RR.Y.H (Figure 5).
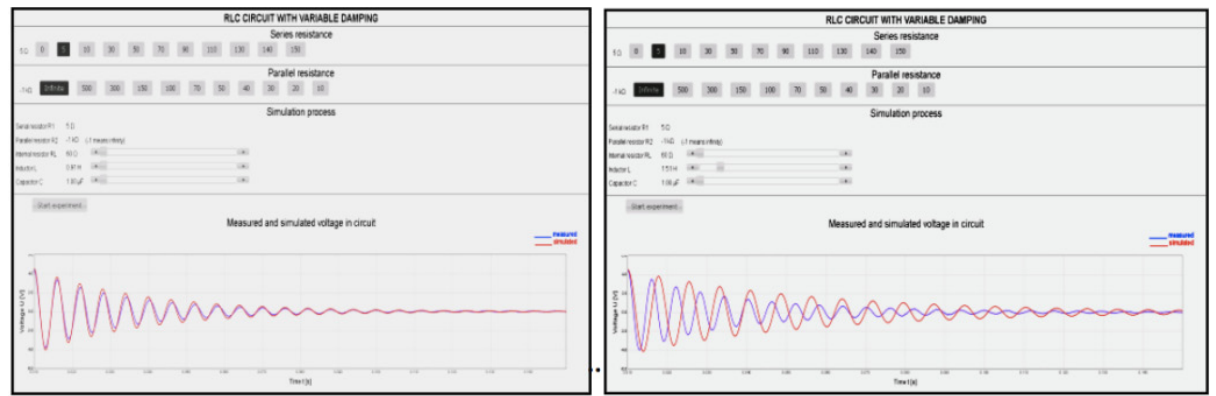

Fig. 4. The time domain response of RLC circuit to step Remote experiment (red) with embedded simulation (blue). Remote experiment data not fitted by simulation data (left) and remote experiment data $100 \%$ fitted by simulation data (right) 


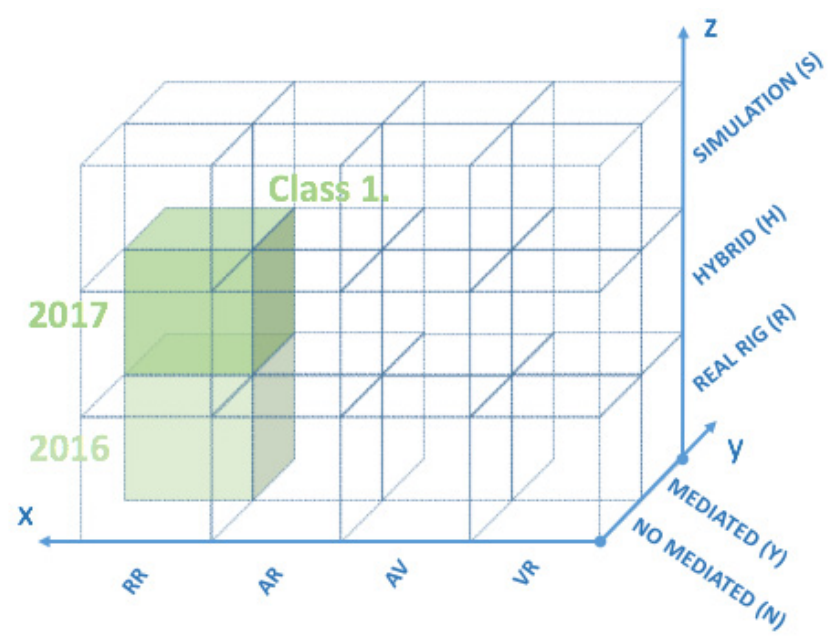

Fig. 5. REMLABNET (version 2016 and 2017) classification

\section{REMLABNET and migration from Class 1 to Class 2}

To enhance user experience with the potential for better understanding of complex phenomena behind remote experiments, one of our current REMLABNET projects focuses on the migration of user interface from Class 1 to Class 2 (with backward compatibility). The complete list of steps requiring this research and the simultaneous development process is shown in Figure 6.

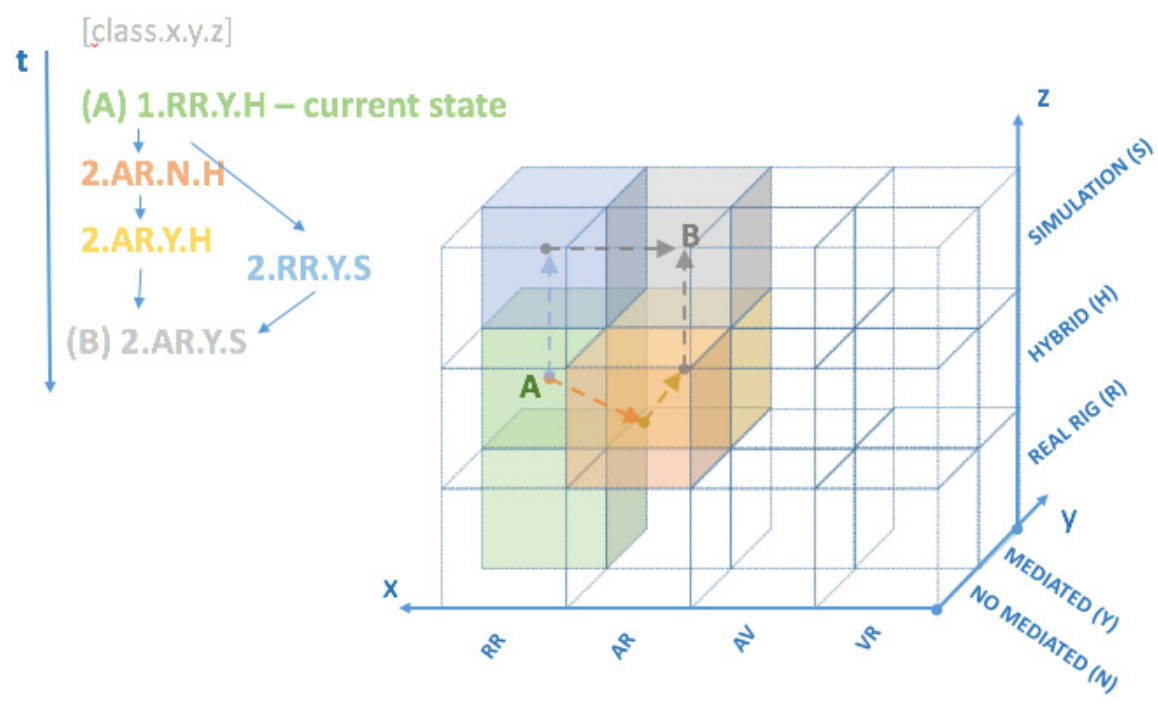

Fig. 6. REMLABNET (version 2017) and the diagram of migration from Class 1 to Class 2 
The transition from stage 1.RR.Y.H to 2.AR.N.H reveals a tremendous potential of AR. We experiment with the mirroring of the rig and related events in the laboratory. A virtual 3-D copy of the real rig can be watched via smart glasses and the rig can be remotely controlled via smart glass sensors. For these objectives, EPSON Moverio BT-300 smart glasses were elected as a mature and affordable device. In this step, a prototype is being developed in Unity $3 \mathrm{D}^{4}$. Unity supports stereoscopic rendering for different VR devices, including the Moverio BT-300 or Samsung Gear VR. In this stage, the control of the experiment is in cooperation with computer - keyboard and mouse (same as in Class 1). However, we are currently researching reasonably advanced handling (e.g. a hand gesture could set a virtual pendulum in motion and this action could be transmitted into the real remote rig). The transition to stage 2.AR.Y.H will bring the mediality of AR. Smart glasses extend the exploitable space where various measurement-related metrics and charts can be displayed. Then a way for remote cooperative learning is opened. Moverio BT-300 (like other similar smart glasses) has a high-resolution camera. Because users have their smart glasses on while measuring, everything that is seen (including the monitor, keyboard, mouse, their hands and virtual elements of the scene) can be streamed to the remote teacher or other students. The following picture (Figure 7) shows the proposed architecture of REMLABNET in this stage.

Full replacement of the real rig via simulation is not possible yet, but mixed embedded simulation is the first step that has to be done before 1.RR.M.S and 2.AR.M.S will be reached. These stages offer a possibility to run the measurement either via the real rig or a fully simulated environment. High acquisition and maintenance costs of redundant rigs (to provide high availability and scaling of experiments) can be significantly reduced. Simulation can make this remote experiment accessible in case the real rig is out of order or being used by another user. This stage will be discussed in more details in future.

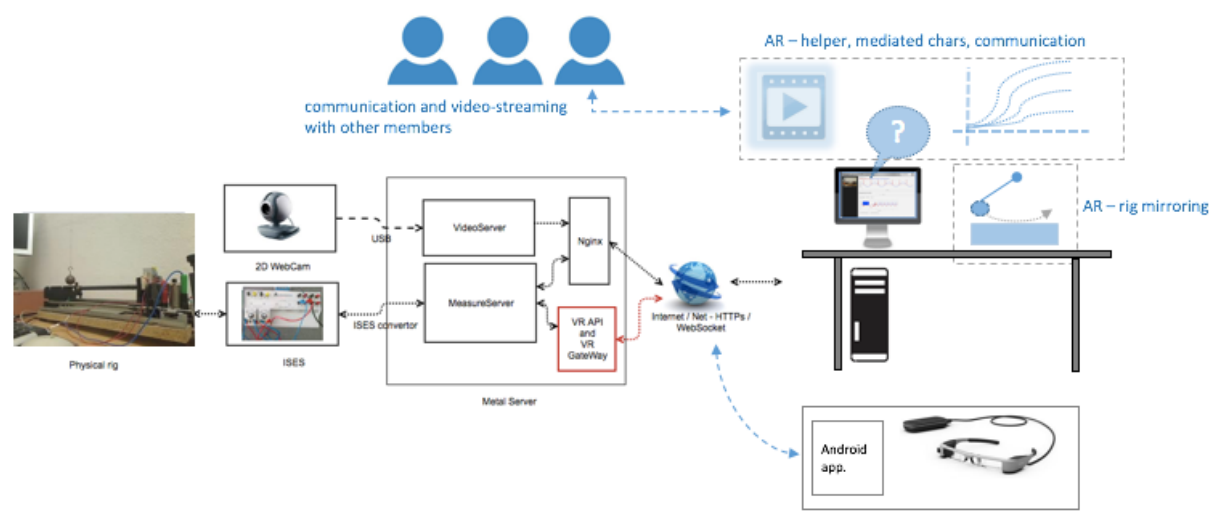

Fig. 7. Proposed schematic representation of a federated Remote Laboratory Management System (RLMS) - REMLABNET(www.remlabnet.eu), extended into 2.AR.Y.H via EPSON Moverio BT-300 and a cooperative education module

\footnotetext{
${ }^{4}$ http://www.unity3d.com
} 


\section{$5 \quad$ Potential of Class 3 and future work}

Class 2 can "take parts of the laboratory to users", but this ability together with the transparency of the smart glass display allows only AR stages to be reached. Class 3, unlike Class 2, provides the capacity to assemble user interface through the whole spectrum of a mixed reality continuum. Due to 3-D $180^{\circ}$ wide-angle cameras, which are able to stream three-dimensional video, it is possible to build a prototype that can "take users into the laboratory". On the other hand, it is possible to offer a severely virtualized and simulated environment. Unfortunately, a few technical limitations do not allow the production of remote experiments for this class on a large scale. The biggest flaws are usually the insufficient resolution of the camera or VR headsets or network limitation between users and the laboratory [5]. Theoretical research on this topic is being conducted. We are developing a prototype which will be able to stream 3-D wide-angle video into VR headset. The combination of Samsung Galaxy S7 and Samsung Galaxy Gear together with $180^{\circ}$ wide-angle 3-D LucidCam is used for prototyping. Our effort is to achieve 3.AR.Y.H, although currently we are in 3.RR.Y.R (Error! Reference source not found..). Nevertheless, our priority is to make significant progress with Class 2 because the combination of the traditional approach (displays, monitors) and lightweight and transparent smart glasses is more natural for users (in spite of the fact that VR headsets will soon provide much more possibilities).

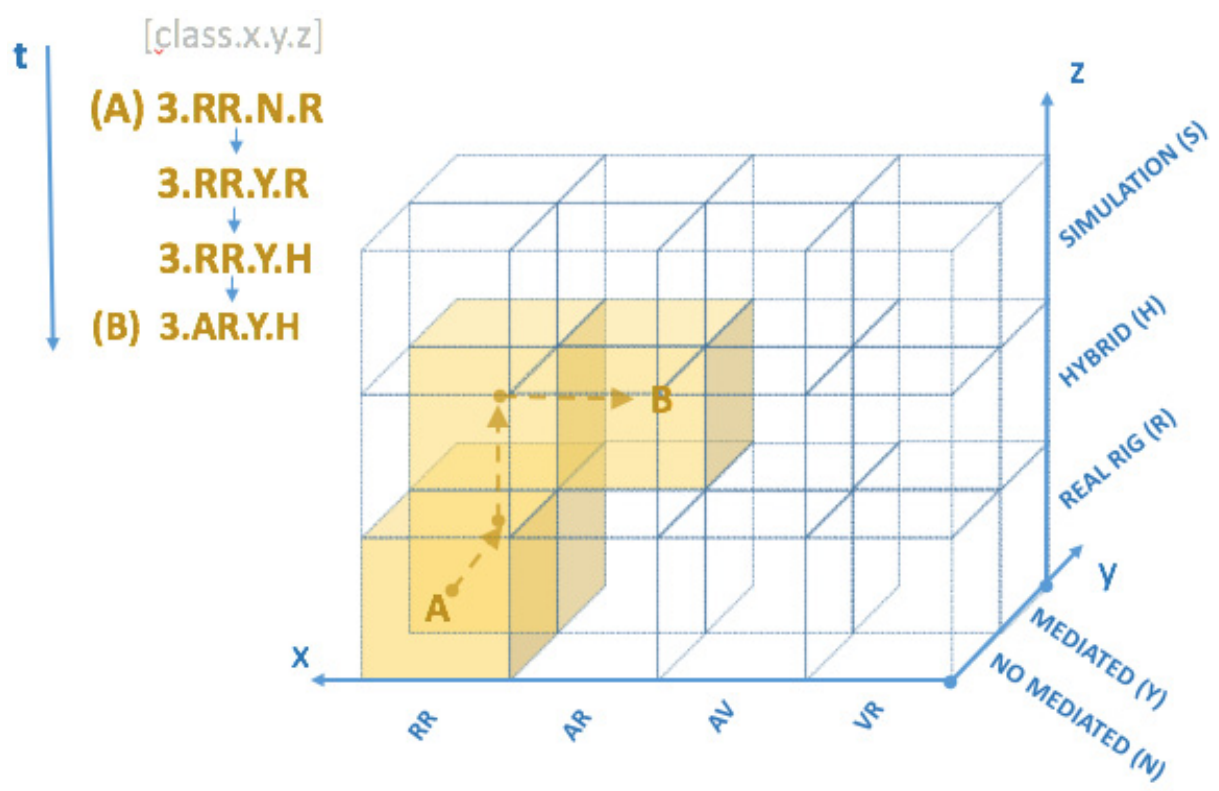

Fig. 8. REMLABNET (version 2017) and the diagram of migration into Class 3 


\section{Acknowledgment}

The support of the project of the Swiss National Science Foundation (SNSF) "SCOPES", No. IZ74Z0_160454 is highly appreciated. The support of the Internal Agency Grant of the Tomas Bata University in Zlin No. IGA/FAI/2017 for PhD students is acknowledged.

\section{$7 \quad$ References}

[1] Schauer, F., Krbecek, M., Beno, M., Gerza. M., Palka, L., Spilakova, P., Komenda, T., Ozvoldova, M., Gerhatova, Z., Tkac, L. (2017). REMLABNET IV - LTI federated remote laboratory management system with embedded multiparameter simulations, in Proc. of REV 2017, New York, USA. pp 340-349. https://doi.org/10.3991/ijoe.v13i10.7802

[2] The Consortium of Remlabnet ( www. remlabnet .eu) is formed by three laboratories in Faculty of Education, Trnava University in Trnava, next, Faculty of Informatics, Tomas Bata University in Zlin and Faculty of Mathematics and Physics, Charles University in Prague.

[3] Poliakov, M., Henke, K., Wuttke, H.D., (2017). The augmented functionality of the physical models of objects of study for remote laboratories, in Proc. of REV 2017, New York, USA. pp 148-157.

[4] Hradio, R., Torre, L., Galan, D., Cabrerizo, F. J., Herrera-Viedma, E., Dormido, S., (2016). Virtual and remote labs in education: A bibliometric analysis. Elsevier: Computers \& Education. pp 14-38. https://doi.org/10.1016/j.compedu.2016.03.010

[5] Smith, M., Maiti, A., Maxwell, A. D., Kist, A. A., (2017). Object Detection Resource Usage within a Remote Real Time Video Stream, in Proc. of REV 2017, New York, USA. pp 792-803.

[6] Schauer, F., Krbecek, M., Beno, P., Gerza, M., Palka, L., Spilaková, P., Tkac, L., (2016). REMLABNET III - federated remote laboratory management system for university and secondary schools, in Proc. 13th International Conference on Remote Engineering and Virtual Instrumentation, REV 2016, Madrid. pp 232-235. https://doi.org/10.1109/REV. 2016.7444471

[7] Orduña, P., Zutin, D. G., Govaerts, S., Zorrozua, I. L., Bailey, P. H., Sancristobal, E., Salzmann, Ch., Rodriguez-Gil, L., DeLong, K. , Gillet, D., Castro, M., Fellow, López-deIpiña, D., García-Zubia, J., (2015). An Extensible Architecture for the Integration of Remote and Virtual Laboratories in Public Learning Tools, IEEE REVISTA IBEROAMERICANA DE TECNOLOGIAS DEL APRENDIZAJE, VOL. 10, NO. 4. pp. 223-233.

[8] Mann, S., (2002). Mediated Reality with implementations for everyday life. presenceconnect.com, the on line companion to the MIT Press journal PRESENCE: Teleoperators and Virtual Environments.

[9] Schauer, F., Gerza, M., Krbecek, M., Ozvoldova, M., (2017). Remote Wave Laboratory real environment for waves mastering, in Proc. of REV 2017, New York, USA. pp 350356.

[10] Aggarwal, V., Kuhlman, R., (2007). Simulated reality, in Electronics, vol. 5, no. 5. pp 1821. 


\section{Authors}

Tomas Komenda is $\mathrm{PhD}$ student with the Tomas Bata University in Zlín, Faculty of Applied Informatics, Nad Stráněmi 4511, Zlín, CZ- 760 05, Czech Republic

Franz Schauer received a M.S. degree in Electronics from the Brno University of Technology in 1963 and his Ph.D. degree in Solid State Physics from Prague University of Technology in 1978. In 1982 he was appointed Associate Professor and in 1988 Professor in Condensed Matter Physics at the Technical Academy in Brno, Czech Republic. In 1993-2002 he was with the Faculty of Chemistry, Brno University of Technology and since then he was with the Polymer Centre of the Faculty of Technology. At present he is with the Tomas Bata University in Zlín, Faculty of Applied Informatics, Czech Republic. His main activities are molecular organic electronics, electronic structure spectroscopy by electrochemical and charge injection methods and computer assisted experiments. He is the author of about 300 papers in both Solid state physics, Education practice and Remote laboratories with about 350 SCI citations.

Article submitted 03 September 2017. Resubmitted 29 December 2017. Final acceptance 05 February 2018. Final version published as submitted by the authors. 